\title{
THE IMPORTANT ROLE OF AMINOETHOXYVINYLGLYCINE (AVG) PRE-HARVEST APPLICATION IN FRUIT HARVEST TIME, FRUIT QUALITY AND MINERAL ELEMENT CONTENTS OF 'AKÇA' (PYRUS COMMUNIS L.)
}

\author{
ÇETINBAŞ, M. \\ Fruit Research Institute, Egirdir, Isparta, Turkey \\ (e-mail: melikecetinbas@gmail.com; phone:+90-246-313-2420; fax:+90-246-313-2425) \\ (Received 19 ${ }^{\text {th }}$ Jun 2018; accepted $7^{\text {th }}$ Aug 2018)
}

\begin{abstract}
Akça' pear is one of the most grown early varieties in Turkey. Although it is of high quality, it forms smaller fruits than other early varieties. In order to investigate the role of aminoethoxyvinylglycine (AVG) application in the harvest time, fruit yield and quality of 'Akça' pears, this study was carried out for 2 years in 2012 and 2013 in an orchard located in Isparta-Egirdir (Turkey) Fruit Research Institute. ReTain plant growth regulator was used for AVG. ReTain containing 15\% AVG was applied to the 'Akça' pear fruits and leaves. Four concentrations of AVG $(0,100,125,150 \mathrm{ppm})$ and three application times [30, 21, 7 days before commercial harvest (DBH)] were evaluated using the field system. Harvest time, fruit yield, fruit size, fruit weight, fruit flesh firmness, titratable acidity (TA), soluble solid contents (SSC), fruit color, ethylene production and respiration rate, and nutritional status of fruits were determined at the harvest time. In addition, the effect of AVG applications on some leaf properties (macro-micro element content, leaf area and chlorophyll) was determined. As a result, 30 and 21 DBH AVG applications delayed the harvest time for 3 days. AVG applications reduced both ethylene production and respiration rate. All AVG applications dramatically increased fruit size (2-16\%) and fruit weight (7-57\%) as well as fruit flesh firmness (1-55\%) of the 'Akça' pear. Given all these results, it is considered that $21 \mathrm{DBH}$ AVG applications at $100 \mathrm{ppm}$ will be suitable for the 'Akça' pear in terms of harvest time and fruit and leaf quality parameters.
\end{abstract}

Keywords: ethylene inhibitor, pear, leave properties, plant growth regulator, nutritional status

\section{Introduction}

The amount of pears produced in the world is 25.798 .644 tons and, with a production amount of 462.336 tons, pear takes the 5th place in Turkey (FAOSTAT, 2018). However, it falls behind other European countries in terms of both yield per decare and exportation. One of the biggest reasons for this is that products with sufficient quality cannot be obtained due to problems related to cultivation techniques. There are many studies conducted in the world for the purpose of increasing fruit quality, and most of them are on plant growth regulators. The most frequently used natural plant growth regulator in the world is the ethylene group (23\%), followed by the auxin group and gibberellins (17\%), respectively. Cytokinin and Dormin are not yet widely used in the world (Barut, 1995). In recent years, AVG (aminoethoxyvinylglycine), an ethylene blocker, has been used to increase pre-harvest fruit fall and fruit quality. AVG is commercially sold worldwide as ReTain ${ }^{\circledR}$ plant growth regulator. ReTain contains $15 \%$ AVG and is a human and environmentally friendly, organic commercial product (Rath and Prentice, 2004) registered in 2001 for use in apples, peaches and nectarine fruits in Australia. Preharvest application of AVG 1-4 weeks prior to harvest inhibits biosynthesis of ethylene and influences abscission, ripening and senescence and reduces quality loss after harvest in many crops (Martinez-Romero et al., 2007; Lurie, 2008; Pech et al., 2012; Kuçuker et al., 2015b; Pasa, et al., 2017). Jobling et al. (2003) found 
that AVG treated plums allowed a delay in harvested timing, and resulted in retaining higher firmness and SSC in comparison with untreated fruit, suggesting that accumulation of soluble solids can take place when fruits are on trees for longer. Dussi et al. (2002) applied 14 DBH 125 and 180 ppm AVG to 'Williams' pears. In this study, AVG had no effect on pre-harvest fall, but both fruit size and fruit hardness increased. It was reported that 21, 14 and $7 \mathrm{DBH} 125 \mathrm{ppm}$ AVG applied to 'Abbe Fetel' pear delayed the harvest for 5 to 15 days (Andreotti et al., 2004). The pear variety 'Akça' is desired by producers and consumers for being an early comer and its quality characteristics. The fruit size of this variety is slightly smaller than other early comers, meaning a decrease in its market price and a disadvantage for producers. The purpose of this study is to determine the appropriate dose and application time of AVG as well as the effectiveness of AVG applications to improve the fruit quality of the 'Akça' pear.

\section{Material and methods}

\section{Plant-chemical materials and experiment design}

The plant material of the study was the 12-year-old Akça/Quince A trees in the pear orchard of Egirdir Fruit Research Institute Directorate Isparta, Turkey) Cultural practices such as irrigation, fertilization, plant protection and pruning were carried out regularly during the trial. Thinning was not performed for the fruits. In our study, ReTain (15\% AVG) plant growth regulator (Valent BioScience Corporation) was used as the chemical material, and AVG doses were calculated based on the active substance. Four doses of AVG - namely, 0 (control), 100, 125 and $150 \mathrm{ppm}$ - were used. These doses were applied to all fruits and leaves of the 'Akça' pear 30, 21, 7 days before harvest (DHB) for two years. All spray solutions contained 0.2\% 'Tween 20' as surfactant. Control trees were sprayed with water + surfactant. A knapsack sprayer was used to apply AVG onto fruits and leaves at the rate of $8 \mathrm{~L} \mathrm{per}$ tree (Fig. 1). The experiment was set up according to the design pattern of 4 repetitive random blocks, and each repetition consisted of 1 tree.
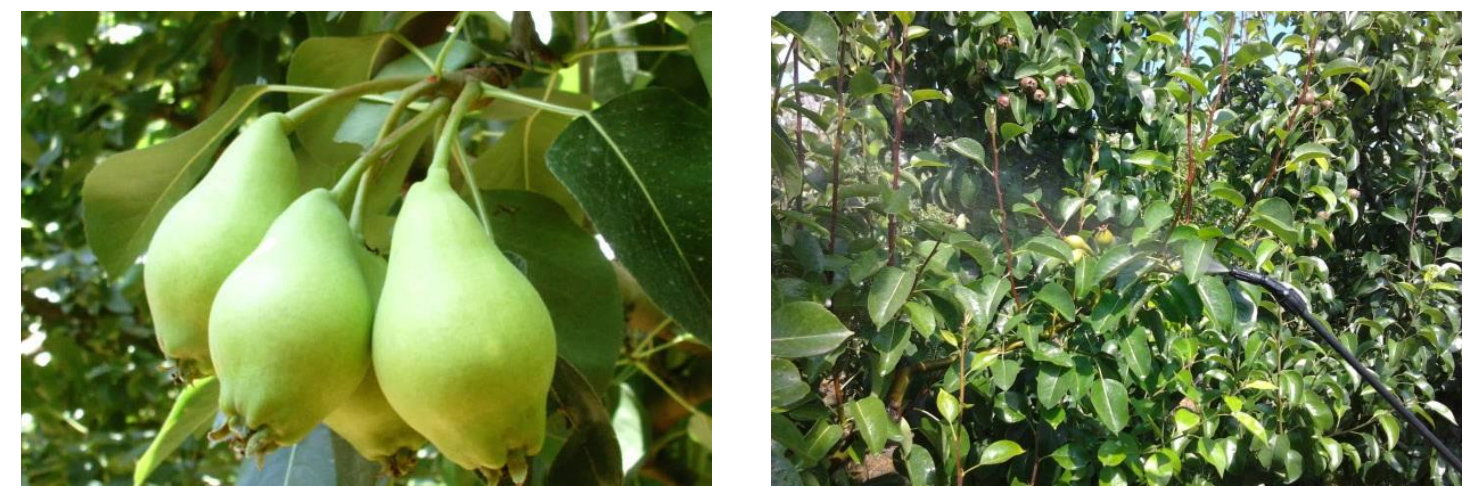

Figure 1. 'Akça' pear and application

\section{Determination of the effect of applications on harvest time}

Determination of harvest time; observation of the fruit size, greenish-yellow coloration of the fruit ground color, hardness of the fruit flesh, and formation of the fruit top color. Harvest was carried out according to these harvest criteria and harvest time 
scales (Ozelkok and Kaynaş, 1995), and the effect of AVG applications on harvest time was determined.

\section{The effect of applications on yield and some fruit quality properties}

Of the trees from each application, yield $\left(\mathrm{kg} \mathrm{tree}^{-1}\right)$ per tree and yield $\left(\mathrm{kg} \mathrm{cm}^{-2}\right)$ per trunk section area were determined. Average values were calculated by measuring the width $(\mathrm{mm})$ and length $(\mathrm{mm})$ of the fruit with the help of a calliper. The average fruit weight $(\mathrm{g})$ of the fruit was calculated by weighing. Flesh firmness measurements were determined as Newton (N) with Lloyd Instruments LF Plus (Ametek, U.K.) texturing equipment. Using a CR-300 Minolta (Japan) color meter and taking measurements from both cheek areas of the fruits, fruit color was determined by CIE L*, $\mathrm{a}^{*}, \mathrm{~b}^{*}, \mathrm{C}^{*}$ and $\mathrm{Hu}^{\circ}$. SSC (with digital refractometer, \%) and TA (titration method, \%) were determined.

\section{The effect of applications on ethylene production and respiratory rate in fruits}

As reported by Dilmaçunal (2009), pears weighing approximately $1 \mathrm{~kg}$ were put into jars and kept at $20 \pm 1{ }^{\circ} \mathrm{C}$ for $24 \mathrm{~h}$ to determine ethylene production $\left(\mu \mathrm{L} \mathrm{kg}^{-1} \mathrm{~h}\right)$ and respiratory rate $\left(\mu \mathrm{L} \mathrm{kg}^{-1} \mathrm{~h}\right)$. Ethylene production and respiratory rate were determined by using gas chromatography.

\section{The effect of applications on fruit and leaf mineral element contents}

\section{$N$ (nitrogen) analysis in fruit and leaf}

In the plant sample burned with a salicylic-sulfuric acid mixture of $2.5 \%$, ammonia formation was obtained in the base environment of the kjeldahl distillation unit. The ammonia formed was then kept in boric acid and titrated with $0.1 \mathrm{~N}$ sulfuric acid. It was calculated as shown in the formula below (Ryan et al., 2001) (Eq. 1).

$$
N \%=\frac{(T-B) \times N \times 1.4}{S}
$$

T: Amount of sulfuric acid consumed for plant sample titration

B: Amount of sulfuric acid consumed for stratum corneum (witness) titration

$\mathrm{N}$ : Normality of sulfuric acid

S: Amount of plant sample used in the analysis

1.4: Coefficient

$P$ (phosphorus), $K$ (potassium), Ca (calcium), $M g$ (magnesium), $C u$ (copper), Fe (iron), Mn (manganese), Zn (zinc), B (boron) analysis in fruit and leaf

Since a considerable amount of boron is lost during wet combustion, dry combustion was performed. Reading was made by ICP (Inductively Coupled Plasma Atomic Emission Spectophometer) (Ryan et al., 2001). The following steps were taken as part of dry combustion: $0.5 \mathrm{~g}$ ground plant material was put into porcelain crucibles, which were then placed into the muffle furnace. Furnace temperature was gradually increased to $550{ }^{\circ} \mathrm{C}$. After reaching $550{ }^{\circ} \mathrm{C}$, combustion continued for $6 \mathrm{~h}$. Following combustion, $5 \mathrm{ml} 2 \mathrm{~N} \mathrm{HCl}$ was added into the cooled porcelain crucibles and the samples were dissolved and stirred with a plastic stick. After 15-20 min, pure water was added until 
reaching $50 \mathrm{ml}$ and thoroughly stirred. 30 min later, it was filtered using a filter paper, and the extract obtained was read by ICP.

\section{The effect of application on leaf area (LA) and total chlorophyll (spad value)}

Total chlorophyll was measured using a portable chlorophyll meter on July 15 (first year) - July 10 (second year) (Minolta SPAD-502, Osaka, Japan). Designed and manufactured based on the principles of Inada (1963), this chlorophyll meter measures the amount of chlorophyll in leaves indirectly. It measures the red and infrared regions in the leaf structure (at $659 \mathrm{~nm}$ and $940 \mathrm{~nm}$ wavelengths, respectively) to determine the relative chlorophyll density. 15 leaf samples were taken in total from four sides of the trees in all applications, and the surface area of one side of each leaf was calculated as a $\mathrm{cm}^{2}$ by a digital planimeter (Koizumi KP-90 N) (Kucukyumuk et al., 2015).

\section{Statistical analysis of results}

Variance analysis was performed with the obtained data, and differences between the treatments were tested by Duncan's multiple range test at P < 0.05 using SSPS (V. 18; Statiscal software, SPSS. Inc., USA) software program. The experiment was based as a completely randomized blocking pattern as four replications and was assigned as one tree for each replication, and was assigned to one tree at each replication with each plot having 20 pear fruits.

\section{Results}

\section{Fruit harvest time and yield}

The harvest was made 2 times a year for both years, and it was seen that the applications affected harvest dates. In the $1^{\text {st }}$ and $2^{\text {nd }}$ years, the control group and the fruits with $7 \mathrm{DBH}$ AVG ripened at the same time, while the fruits with 30 and $21 \mathrm{DBH}$ AVG ripened later. After reaching maturity for the first harvest, the control and the fruits with 7 DBH AVG were harvested on July 14 in the first year and on July 6 in the second year. On the other hand, other fruits with 30 and $21 \mathrm{DBH}$ AVG were harvested on July 18 in the first year and on July 9 in the second year. It was determined that AVG applications (except for $7 \mathrm{DBH}$ ) delayed the harvest maturity of Akça pear for up to 3-4 days. AVG applications did not have any statistically significant effect on yield per tree or yield per trunk section in both years (Table 1).

Table 1. The effect of AVG treatments on yield in the 'Akça' pear, 2012 and 2013

\begin{tabular}{c|c|c|c|c|c}
\hline \multirow{2}{*}{ Application time $^{\mathbf{1}}$} & \multirow{2}{*}{$\begin{array}{c}\text { AVG doses } \\
(\mathbf{p p m})\end{array}$} & \multicolumn{2}{|c|}{ Yield $\left(\mathbf{k g ~ t r e e}^{\mathbf{- 1}}\right)$} & \multicolumn{2}{c}{ Yield $\left.\mathbf{k g ~ c m}^{-\mathbf{2}}\right)$} \\
\cline { 3 - 6 } & & I. Year & II. Year & I. Year & II. Year \\
\hline \multirow{3}{*}{$30 \mathrm{~d}$} & 0 & 8.52 & 12.78 & 0.02 & 0.29 \\
& 100 & 8.93 & 13.40 & 0.02 & 0.28 \\
& 125 & 9.27 & 13.90 & 0.02 & 0.28 \\
& 150 & 9.60 & 14.40 & 0.03 & 0.32 \\
\hline \multirow{3}{*}{$21 \mathrm{~d}$} & 0 & 8.68 & 12.78 & 0.03 & 0.29 \\
& 100 & 10.33 & 15.50 & 0.03 & 0.33 \\
& 125 & 10.73 & 16.10 & 0.03 & 0.36 \\
& 150 & 10.33 & 15.50 & 0.03 & 0.40 \\
\hline
\end{tabular}




\begin{tabular}{c|c|c|c|c|c}
\hline & 0 & 8.22 & 12.78 & 0.02 & 0.29 \\
$7 \mathrm{~d}$ & 100 & 13.20 & 19.80 & 0.04 & 0.48 \\
& 125 & 10.70 & 16.05 & 0.03 & 0.37 \\
& 150 & 9.80 & 14.70 & 0.03 & 0.36 \\
\hline Time means & & & & & \\
30 & & 9.08 & 13.62 & 0.02 & 0.29 \\
21 & & 10.02 & 14.97 & 0.03 & 0.34 \\
7 & & 10.48 & 15.83 & 0.03 & 0.37 \\
\hline & Dose means & & & & \\
& 0 & 8.47 & 12.78 & 0.02 & 0.29 \\
& 100 & 10.82 & 16.23 & 0.03 & 0.36 \\
& 125 & 10.23 & 15.35 & 0.03 & 0.34 \\
$P$ values & 150 & 9.91 & 14.87 & 0.03 & 0.36 \\
Time (T) & & & & & \\
AVG dose (D) & & 0.275 & 0.251 & 0.267 & 0.096 \\
T x D & 0.702 & 0.748 & 0.956 & 0.864 \\
\hline
\end{tabular}

${ }^{1}$ Days before harvest (DBH)

\section{Fruit quality}

As a result of the variance analysis performed in both application years, the time $\mathrm{x}$ dose interactions were found statistically significant $(\mathrm{p}<0.05)$ in terms of fruit width (Table 2). In both years, the AVG applications increased fruit width. The applications with the highest fruit width in the first and second years were $21 \mathrm{DBH} 100 \mathrm{ppm}$ AVG (48.32 mm) and $30 \mathrm{DBH} 100 \mathrm{ppm}$ AVG (49.54 mm), respectively (Table 2). The effect of AVG applications on fruit length was statistically significant $(\mathrm{p}<0.05)$ only in the first year as time $\mathrm{x}$ dose interaction (Table 2). Just like in fruit width, the applications with the highest fruit length were $21 \mathrm{DBH} 100 \mathrm{ppm}$ AVG ( $1^{\text {st }}$ year). On the other hand, the lowest fruit length was observed in the control fruits with 7 DBH AVG application ( $1^{\text {st }}$ year) (Table 2$)$. Fruit weight increased with the applications in both years, and the time $\mathrm{x}$ dose interaction was found to be statistically significant in the analysis of variance $(\mathrm{p}<0.05)$. The heaviest fruits were obtained from 30 and $21 \mathrm{DBH} 100 \mathrm{ppm}$ AVG applications. The least heavy fruits were again obtained from the controls (Table 2).

Table 2. The effect of AVG treatments on fruit width, length and weight in the 'Akça' pear, 2012 and 2013

\begin{tabular}{c|c|c|c|c|c|c|c}
\hline \multirow{2}{*}{ Application time $^{\mathbf{1}}$} & \multirow{2}{*}{$\begin{array}{c}\text { AVG doses } \\
\text { (ppm) }\end{array}$} & \multicolumn{2}{|c|}{ Fruit width $(\mathbf{m m})$} & \multicolumn{2}{c|}{ Fruit length $(\mathbf{m m})$} & \multicolumn{2}{c}{ Fruit weight (g) } \\
\cline { 3 - 8 } & I. Year & II. Year & I. Year & II. Year & I. Year & II. Year \\
\hline \multirow{3}{*}{$30 \mathrm{~d}$} & 0 & $45.02 \mathrm{c}-\mathrm{e}^{*}$ & $42.53 \mathrm{~b}^{*}$ & $56.79 \mathrm{e}-\mathrm{g}^{*}$ & 79.55 & $53.17 \mathrm{de}^{*}$ & $47.86 \mathrm{c}^{*}$ \\
& 100 & $47.81 \mathrm{ab}$ & $49.54 \mathrm{a}$ & $63.32 \mathrm{bc}$ & 69.64 & $68.07 \mathrm{a}$ & $71.28 \mathrm{a}$ \\
& 125 & $47.54 \mathrm{ab}$ & $48.75 \mathrm{a}$ & $64.72 \mathrm{~b}$ & 68.89 & $64.39 \mathrm{ab}$ & $70.42 \mathrm{a}$ \\
& 150 & $47.07 \mathrm{a}-\mathrm{d}$ & $48.01 \mathrm{a}$ & $62.81 \mathrm{~b}-\mathrm{d}$ & 68.08 & $60.27 \mathrm{bc}$ & $67.00 \mathrm{a}$ \\
\hline
\end{tabular}




\begin{tabular}{c|c|c|c|c|c|c|c}
\hline & 0 & $45.74 \mathrm{~b}-\mathrm{e}$ & $42.30 \mathrm{~b}$ & $55.97 \mathrm{fg}$ & 60.81 & $53.71 \mathrm{c}-\mathrm{e}$ & $46.50 \mathrm{c}$ \\
$21 \mathrm{~d}$ & 100 & $48.32 \mathrm{a}$ & $49.23 \mathrm{a}$ & $68.61 \mathrm{a}$ & 70.64 & $67.10 \mathrm{a}$ & $73.26 \mathrm{a}$ \\
& 125 & $47.62 \mathrm{ab}$ & $48.44 \mathrm{a}$ & $65.68 \mathrm{ab}$ & 66.09 & $64.06 \mathrm{ab}$ & $67.41 \mathrm{a}$ \\
& 150 & $46.31 \mathrm{a}-\mathrm{d}$ & $48.76 \mathrm{a}$ & $62.16 \mathrm{~b}-\mathrm{d}$ & 69.99 & $57.17 \mathrm{~cd}$ & $71.77 \mathrm{a}$ \\
\hline \multirow{3}{*}{$7 \mathrm{~d}$} & 0 & $44.05 \mathrm{e}$ & $42.79 \mathrm{~b}$ & $53.78 \mathrm{~g}$ & 63.30 & $49.01 \mathrm{e}$ & $49.17 \mathrm{c}$ \\
& 100 & $45.89 \mathrm{~b}-\mathrm{e}$ & $48.38 \mathrm{a}$ & $60.09 \mathrm{c}-\mathrm{e}$ & 65.58 & $55.84 \mathrm{~cd}$ & $67.43 \mathrm{a}$ \\
& 125 & $44.97 \mathrm{de}$ & $48.99 \mathrm{a}$ & $59.50 \mathrm{~d}-\mathrm{f}$ & 65.14 & $52.62 \mathrm{de}$ & $68.20 \mathrm{a}$ \\
& 150 & $47.12 \mathrm{a}-\mathrm{c}$ & $44.10 \mathrm{~b}$ & $60.97 \mathrm{~cd}$ & 56.09 & $60.14 \mathrm{bc}$ & $61.12 \mathrm{~b}$ \\
\hline Time means & & & & & & & \\
30 & & 46.86 & 47.21 & 61.91 & 71.54 & 61.48 & 64.14 \\
21 & & 47.00 & 47.18 & 63.11 & 66.88 & 60.51 & 64.74 \\
7 & & 45.51 & 46.07 & 58.59 & 62.53 & 54.40 & 61.48 \\
\hline & Doses & & & & & & \\
& means & & & & & & \\
& 0 & 44.94 & 42.54 & 55.52 & 67.89 & 51.97 & 47.84 \\
& 100 & 47.34 & 49.05 & 64.01 & 68.62 & 63.67 & 70.66 \\
& 125 & 46.71 & 48.73 & 63.30 & 66.71 & 60.36 & 68.68 \\
\hline Time (T) & 150 & 46.83 & 46.96 & 61.98 & 64.72 & 59.19 & 66.63 \\
\hline T & & & & & & & \\
\hline
\end{tabular}

${ }^{1}$ Days before harvest (DBH)

*Each column, values followed by the same letter are not significantly different at $p<0.05$ level according to Duncan's multiple range test

The time $\mathrm{x}$ dose interaction of AVG applications was statistically significant $(p<0.05)$ in terms of fruit flesh firmness in both years. In both years, fruit flesh firmness increased with the applications, and the fruit flesh firmness of the applications of $21 \mathrm{DBH}$ was higher than others. $21 \mathrm{DBH} 100 \mathrm{ppm}$ AVG in the first year and $30 \mathrm{DBH}$ $150 \mathrm{ppm}$ AVG in the second year gave the hardest fruits (Table 3).

Table 3. The effect of AVG treatments on fruit flesh firmness, SSC and TA in the 'Akça' pear, 2012 and 2013

\begin{tabular}{c|c|c|c|c|c|c|c}
\hline \multirow{2}{*}{ Application time1 $^{1}$} & AVG doses & \multicolumn{2}{|c|}{ Firmness (N) } & \multicolumn{2}{c|}{ SSC (\%) } & \multicolumn{2}{c}{ TA (\%) } \\
\cline { 3 - 8 } & $(\mathbf{p p m})$ & I. Year & II. Year & I. Year & II. Year & I. Year & II. Year \\
\hline \multirow{3}{*}{$30 \mathrm{~d}$} & 0 & $88.99 \mathrm{c}^{*}$ & $82.22 \mathrm{~b}^{*}$ & $12.73 \mathrm{~b}^{*}$ & 12.13 & $0.17 \mathrm{a}-\mathrm{d}^{*}$ & $0.17 \mathrm{~b}-\mathrm{d}^{*}$ \\
& 100 & $89.77 \mathrm{bc}$ & $94.14 \mathrm{ab}$ & $11.23 \mathrm{~d}$ & 12.83 & $0.17 \mathrm{a}-\mathrm{d}$ & $0.17 \mathrm{~b}-\mathrm{d}$ \\
& 125 & $92.33 \mathrm{~b}$ & $97.00 \mathrm{ab}$ & $11.33 \mathrm{~d}$ & 12.27 & $0.15 \mathrm{~d}$ & $0.16 \mathrm{~d}$ \\
& 150 & $90.93 \mathrm{~b}$ & $104.13 \mathrm{a}$ & $11.87 \mathrm{~cd}$ & 12.33 & $0.17 \mathrm{a}-\mathrm{d}$ & $0.17 \mathrm{~b}-\mathrm{d}$ \\
\hline \multirow{3}{*}{$21 \mathrm{~d}$} & 0 & $86.30 \mathrm{c}$ & $83.33 \mathrm{~b}$ & $12.80 \mathrm{ab}$ & 12.27 & $0.17 \mathrm{a}-\mathrm{d}$ & $0.16 \mathrm{~d}$ \\
& 100 & $95.15 \mathrm{a}$ & $93.83 \mathrm{ab}$ & $11.90 \mathrm{~cd}$ & 12.00 & $0.19 \mathrm{a}$ & $0.19 \mathrm{a}$ \\
& 125 & $93.00 \mathrm{a}$ & $96.95 \mathrm{ab}$ & $12.53 \mathrm{bc}$ & 12.07 & $0.17 \mathrm{a}-\mathrm{d}$ & $0.16 \mathrm{~d}$ \\
& 150 & $95.02 \mathrm{a}$ & $100.08 \mathrm{ab}$ & $12.60 \mathrm{~b}$ & 12.23 & $0.16 \mathrm{~b}-\mathrm{d}$ & $0.17 \mathrm{~b}-\mathrm{d}$ \\
\hline
\end{tabular}




\begin{tabular}{c|c|c|c|c|c|c|c}
\hline & 0 & $89.83 \mathrm{bc}$ & $63.97 \mathrm{c}$ & $12.90 \mathrm{ab}$ & 12.33 & $0.17 \mathrm{a}-\mathrm{d}$ & $0.18 \mathrm{ab}$ \\
$7 \mathrm{~d}$ & 100 & $91.35 \mathrm{~b}$ & $89.60 \mathrm{ab}$ & $12.83 \mathrm{ab}$ & 12.37 & $0.16 \mathrm{~cd}$ & $0.17 \mathrm{~b}-\mathrm{d}$ \\
& 125 & $91.98 \mathrm{~b}$ & $87.21 \mathrm{ab}$ & $13.47 \mathrm{a}$ & 11.80 & $0.17 \mathrm{a}-\mathrm{c}$ & $0.18 \mathrm{ab}$ \\
& 150 & $87.39 \mathrm{c}$ & $99.37 \mathrm{ab}$ & $12.87 \mathrm{ab}$ & 12.67 & $0.18 \mathrm{ab}$ & $0.18 \mathrm{ab}$ \\
\hline Time means & & & & & & & \\
30 & & 90.51 & 94.37 & 11.79 & 12.39 & 0.17 & 0.01 \\
21 & & 92.37 & 93.55 & 12.46 & 12.14 & 0.17 & 0.01 \\
7 & & 90.14 & 85.04 & 13.02 & 12.29 & 0.18 & 0.02 \\
\hline & Dose means & & & & & & \\
& 0 & 88.37 & 76.51 & 12.81 & 12.24 & 0.17 & 0.01 \\
& 100 & 92.09 & 92.52 & 11.99 & 12.40 & 0.17 & 0.01 \\
& 125 & 92.44 & 93.72 & 12.44 & 12.04 & 0.17 & 0.01 \\
\hline Time (T) & 150 & 91.11 & 101.19 & 12.44 & 12.41 & 0.17 & 0.01 \\
\hline AVG Dose (D) & & & & & & & \\
T x D & & 0.406 & 0.176 & 0.000 & 0.371 & 0.375 & 0.001 \\
\hline
\end{tabular}

${ }^{1}$ Days before harvest (DBH)

*Each column, values followed by the same letter are not significantly different at $\mathrm{p}<0.05$ level according to Duncan's multiple range test

In the first year, the applications increased the amount of SSC, and the time $\mathrm{x}$ dose interaction was found to be significant $(\mathrm{p}<0.05)$ as a result of the variance analysis. In the second year, however, the applications were statistically insignificant. In the first year, the highest SSC (13.47\%) was found in 7 DBH 125 ppm AVG (Table 3). The time $x$ dose interaction was statistically significant $(p<0.05)$ in terms of TA in both years, and the highest TA was obtained from 21 DBH 100 ppm AVG (Table 3).

In the first year, the effect of AVG applications on fruit color values $\left(L^{*}, a^{*}, b^{*}, C^{*}\right.$, $\mathrm{h}^{\circ}$ ) was statistically significant $(\mathrm{p}<0.05)$ in time $\mathrm{x}$ dose interactions (Table 4).

Table 4. The effect of AVG treatments on fruit color $\left(L^{*}, a^{*}, b^{*}, C^{*}, h^{\circ}\right)$ in the 'Akça' pear, 2012

\begin{tabular}{c|c|c|c|c|c|c}
\hline Application time $^{\mathbf{1}}$ & $\begin{array}{c}\text { AVG doses } \\
\text { (ppm) }\end{array}$ & $\mathbf{L}^{*}$ & $\mathbf{a}^{*}$ & $\mathbf{b}^{*}$ & $\mathbf{C}^{*}$ & $\mathbf{h}^{\circ}$ \\
\hline \multirow{3}{*}{$30 \mathrm{~d}$} & 0 & $72.79 \mathrm{a}^{*}$ & $-19.11 \mathrm{a}^{*}$ & $48.47 \mathrm{ab} *$ & $52.16 \mathrm{bc} *$ & $111.54 \mathrm{f}^{*}$ \\
& 100 & $70.43 \mathrm{de}$ & $-21.19 \mathrm{~cd}$ & $48.01 \mathrm{bc}$ & $52.52 \mathrm{bc}$ & $113.82 \mathrm{~b}-\mathrm{d}$ \\
& 125 & $69.19 \mathrm{f}$ & $-21.84 \mathrm{de}$ & $47.32 \mathrm{~d}$ & $52.13 \mathrm{bc}$ & $114.79 \mathrm{ab}$ \\
& 150 & $69.92 \mathrm{~d}-\mathrm{f}$ & $-21.59 \mathrm{c}-\mathrm{e}$ & $47.37 \mathrm{~d}$ & $52.07 \mathrm{c}$ & $114.51 \mathrm{a}-\mathrm{c}$ \\
\hline \multirow{3}{*}{$21 \mathrm{~d}$} & 0 & $72.53 \mathrm{ab}$ & $-19.18 \mathrm{a}$ & $48.60 \mathrm{ab}$ & $52.32 \mathrm{bc}$ & $111.55 \mathrm{f}$ \\
& 100 & $67.96 \mathrm{~g}$ & $-22.21 \mathrm{e}$ & $47.16 \mathrm{~d}$ & $52.14 \mathrm{bc}$ & $115.23 \mathrm{a}$ \\
& 125 & $69.54 \mathrm{ef}$ & $-21.82 \mathrm{de}$ & $47.58 \mathrm{~cd}$ & $52.35 \mathrm{bc}$ & $114.64 \mathrm{a}-\mathrm{c}$ \\
& 150 & $70.48 \mathrm{de}$ & $-21.30 \mathrm{c}-\mathrm{e}$ & $48.23 \mathrm{a}-\mathrm{c}$ & $52.74 \mathrm{ab}$ & $113.84 \mathrm{~b}-\mathrm{d}$ \\
\hline \multirow{3}{*}{$7 \mathrm{~d}$} & 0 & $71.62 \mathrm{bc}$ & $-20.15 \mathrm{~b}$ & $48.18 \mathrm{bc}$ & $52.26 \mathrm{bc}$ & $112.72 \mathrm{e}$ \\
& 100 & $70.65 \mathrm{c}-\mathrm{e}$ & $-21.07 \mathrm{~b}-\mathrm{d}$ & $48.85 \mathrm{a}$ & $53.24 \mathrm{a}$ & $113.35 \mathrm{de}$ \\
& 125 & $70.52 \mathrm{de}$ & $-20.82 \mathrm{bc}$ & $48.43 \mathrm{ab}$ & $52.76 \mathrm{ab}$ & $113.27 \mathrm{de}$ \\
& 150 & $70.71 \mathrm{~cd}$ & $-20.98 \mathrm{~b}-\mathrm{d}$ & $48.00 \mathrm{bc}$ & $52.41 \mathrm{bc}$ & $113.63 \mathrm{c}-\mathrm{e}$ \\
\hline
\end{tabular}




\begin{tabular}{c|c|c|c|c|c|c}
\hline Time means & & 70.58 & -20.93 & 47.79 & 52.22 & 113.66 \\
30 & & 70.13 & -21.13 & 47.89 & 52.39 & 113.82 \\
21 & & 70.87 & -20.75 & 48.37 & 52.67 & 113.24 \\
7 & & & & & \\
& Dose means & & & & & \\
& 0 & 72.31 & -19.48 & 48.42 & 52.24 & 111.94 \\
& 100 & 69.68 & -21.49 & 48.01 & 52.63 & 114.13 \\
& 125 & 69.75 & -21.49 & 47.78 & 52.41 & 114.23 \\
$P$ values & 150 & 70.37 & -21.29 & 47.87 & 52.41 & 113.99 \\
Time (T) & & & & & & \\
AVG Dose (D) & & 0.431 & 0.706 & 0.041 & 0.032 & 0.517 \\
T x D & 0.902 & 0.965 & 0.758 & 0.670 & 0.218 \\
\hline
\end{tabular}

${ }^{1}$ Days before harvest (DBH)

*Each column, values followed by the same letter are not significantly different at $p<0.05$ level according to Duncan's multiple range test

The values of $\mathrm{L}^{*}$, which indicates brightness, decreased with the AVG applications. The lowest $\mathrm{L}^{*}$ was obtained from $21 \mathrm{DBH} 100$ ppm AVG (67.96). The values of $\mathrm{a}^{*}$, which indicates redness, also decreased with the applications, and the lowest value was obtained from $21 \mathrm{DBH} 100 \mathrm{ppm}$ AVG, which also gave the value of $\mathrm{b}^{*}$ indicating yellowness. On the other hand, the highest value of $b^{*}$ was obtained from $7 \mathrm{DBH}$ $100 \mathrm{ppm}$ AVG. As in $\mathrm{b}^{*}$ values, the highest $\mathrm{C}^{*}$ value was produced by $7 \mathrm{DBH} 100 \mathrm{ppm}$ AVG. The hue values of AVG-applied fruits were higher than those of the control fruits, and the highest $\mathrm{h}^{\circ}$ value was produced by $21 \mathrm{DBH} 100 \mathrm{ppm}$ AVG (Table 4). In the second year of the project, the time $x$ dose interactions of $L^{*}, a^{*}, h^{\circ}$ values were found to be statistically significant $(\mathrm{p}<0.05)$. $\mathrm{L}^{*}$ and $\mathrm{a}^{*}$ values decreased with the AVG applications, and the lowest $\mathrm{L}^{*}(67.78)$ and $\mathrm{a}^{*}$ (22.58) values were observed in $21 \mathrm{DBH} 150 \mathrm{ppm}$ AVG. It was found that the $\mathrm{h}^{\circ}$ values of the AVG applications were higher than the control fruits, and the highest $\mathrm{h}^{\circ}$ value was obtained from $21 \mathrm{DBH}$ 125 ppm AVG (Table 5).

Table 5. The effect of AVG treatments on fruit color $\left(L^{*}, a^{*}, b^{*}, C^{*}, h^{\circ}\right)$ in the 'Akça' pear, 2013

\begin{tabular}{c|c|c|c|c|c|c}
\hline Application time & $\begin{array}{c}\text { AVG doses } \\
(\mathbf{p p m})\end{array}$ & $\mathbf{L}^{*}$ & $\mathbf{a}^{*}$ & $\mathbf{b}^{*}$ & $\mathbf{C}^{*}$ & $\mathbf{h}^{\circ}$ \\
\hline \multirow{3}{*}{$30 \mathrm{~d}$} & 0 & $73.90 \mathrm{a}^{*}$ & $-19.66 \mathrm{ab}$ & 48.36 & 52.32 & $112.12 \mathrm{~b}-\mathrm{d}^{*}$ \\
& 100 & $69.17 \mathrm{~b}$ & $-22.32 \mathrm{c}$ & 47.46 & 52.46 & $115.19 \mathrm{a}$ \\
& 125 & $70.08 \mathrm{~b}$ & $-22.00 \mathrm{c}$ & 47.53 & 52.38 & $114.85 \mathrm{a}$ \\
& 150 & $69.96 \mathrm{~b}$ & $-22.49 \mathrm{c}$ & 47.19 & 52.28 & $115.49 \mathrm{a}$ \\
\hline \multirow{3}{*}{$21 \mathrm{~d}$} & 0 & $73.46 \mathrm{a}$ & $-19.35 \mathrm{ab}$ & 48.60 & 52.48 & $111.69 \mathrm{~cd}$ \\
& 100 & $68.95 \mathrm{~b}$ & $-22.21 \mathrm{c}$ & 47.93 & 52.83 & $114.87 \mathrm{a}$ \\
& 125 & $69.96 \mathrm{~b}$ & $-22.01 \mathrm{c}$ & 47.69 & 52.54 & $114.78 \mathrm{a}$ \\
& 150 & $67.78 \mathrm{~b}$ & $-22.58 \mathrm{c}$ & 47.10 & 52.24 & $115.62 \mathrm{a}$ \\
\hline
\end{tabular}




\begin{tabular}{c|c|c|c|c|c|c}
\hline & 0 & $74.50 \mathrm{a}$ & $-18.68 \mathrm{a}$ & 48.74 & 52.30 & $110.94 \mathrm{~d}$ \\
$7 \mathrm{~d}$ & 100 & $69.68 \mathrm{~b}$ & $-21.82 \mathrm{c}$ & 48.42 & 53.13 & $114.29 \mathrm{ab}$ \\
& 125 & $70.73 \mathrm{~b}$ & $-21.07 \mathrm{bc}$ & 48.19 & 52.66 & $113.63 \mathrm{a}-\mathrm{c}$ \\
& 150 & $69.93 \mathrm{~b}$ & $-21.30 \mathrm{bc}$ & 47.72 & 52.29 & $114.07 \mathrm{a}-\mathrm{c}$ \\
\hline Time means & & & & & & \\
30 & & 70.78 & -21.62 & 47.63 & 52.36 & 114.41 \\
21 & & 70.04 & -21.54 & 47.83 & 52.52 & 114.24 \\
7 & & 71.21 & -20.72 & 48.27 & 52.60 & 113.23 \\
\hline & Dose means & & & & & \\
& 0 & 73.95 & -19.23 & 48.56 & 52.37 & 111.58 \\
& 100 & 69.27 & -22.12 & 47.93 & 52.81 & 114.78 \\
& 125 & 70.25 & -21.69 & 47.80 & 52.53 & 114.42 \\
$P$ values & 150 & 69.22 & -22.12 & 47.34 & 52.27 & 115.06 \\
Time (T) & & & & & & \\
AVG Dose (D) & & 0.499 & 0.328 & 0.115 & 0.505 & 0.266 \\
T x D & 0.804 & 0.843 & 0.471 & 0.122 & 0.883 \\
\hline
\end{tabular}

${ }^{1}$ Days before harvest (DBH)

*Each column, values followed by the same letter are not significantly different at $p<0.05$ level according to Duncan's multiple range test

\section{Ethylene production and respiration rate}

In the first year, ethylene production could not be determined. In terms of respiration rate, the time $x$ dose interaction was found statistically significant $(p<0.05)$. In the second year, however, the effect of the applications on both ethylene production and respiration rate was found to be statistically significant $(\mathrm{p}<0.05)$ in terms of time $\mathrm{x}$ dose interaction (Table 6). All AVG applications slowed both ethylene production $\left(\mu \mathrm{Lg}^{-1} \mathrm{~h}\right)$ and respiratory rate $\left(\mu \mathrm{L} \mathrm{kg}^{-1} \mathrm{~h}\right)$ in the fruit. The lowest ethylene production was $0.15 \mu \mathrm{L} \mathrm{kg}^{-1} \mathrm{~h}$ and the lowest respiration rate was $12.32 \mu \mathrm{L} \mathrm{kg}^{-1} \mathrm{~h}$ obtained from $21 \mathrm{DBH} 150$ ppm AVG (Table 6).

Table 6. The effect of AVG treatments on ethylene production and respiration rate in the 'Akça' pear, 2012 and 2013

\begin{tabular}{c|c|c|c|c|c}
\hline \multirow{2}{*}{$\begin{array}{c}\text { Application time } \\
\end{array}$} & $\begin{array}{c}\text { AVG doses } \\
(\mathbf{p p m})\end{array}$ & \multicolumn{2}{|c|}{$\begin{array}{c}\text { Ethylene production } \\
\left(\boldsymbol{\mu} \mathbf{~ k g}^{-1} \mathbf{h}\right)\end{array}$} & \multicolumn{2}{c}{$\begin{array}{c}\text { Respiration rate } \\
\left(\boldsymbol{\mu} \mathbf{~ k g}^{-1} \mathbf{h}\right)\end{array}$} \\
\cline { 3 - 6 } & & I. Year & II. Year & I. Year & II. Year \\
\hline \multirow{3}{*}{$30 \mathrm{~d}$} & 0 & - & $3.54 \mathrm{a}^{*}$ & $15.41 \mathrm{a}-\mathrm{c}^{*}$ & $28.16 \mathrm{a}^{*}$ \\
& 100 & - & $0.86 \mathrm{~d}-\mathrm{f}$ & $15.13 \mathrm{bc}$ & $18.22 \mathrm{~b}-\mathrm{d}$ \\
& 125 & - & $1.12 \mathrm{c}-\mathrm{f}$ & $15.87 \mathrm{a}-\mathrm{c}$ & $15.06 \mathrm{~cd}$ \\
& 150 & - & $0.28 \mathrm{ef}$ & $15.96 \mathrm{a}-\mathrm{c}$ & $13.80 \mathrm{~cd}$ \\
\hline \multirow{3}{*}{$21 \mathrm{~d}$} & 0 & - & $2.98 \mathrm{ab}$ & $16.72 \mathrm{ab}$ & $27.38 \mathrm{a}$ \\
& 100 & - & $0.56 \mathrm{~d}-\mathrm{f}$ & $16.06 \mathrm{a}-\mathrm{c}$ & $16.55 \mathrm{~b}-\mathrm{d}$ \\
& 125 & - & $0.82 \mathrm{~d}-\mathrm{f}$ & $14.61 \mathrm{c}$ & $19.08 \mathrm{~b}-\mathrm{d}$ \\
& 150 & - & $0.15 \mathrm{f}$ & $15.66 \mathrm{a}-\mathrm{c}$ & $12.32 \mathrm{~d}$ \\
\hline
\end{tabular}




\begin{tabular}{|c|c|c|c|c|c|}
\hline $7 \mathrm{~d}$ & $\begin{array}{c}0 \\
100 \\
125 \\
150 \\
\end{array}$ & $\begin{array}{l}- \\
- \\
- \\
-\end{array}$ & $\begin{array}{l}2.09 \mathrm{bc} \\
2.02 \mathrm{bc} \\
1.56 \mathrm{~cd} \\
1.23 \mathrm{c}-\mathrm{e}\end{array}$ & $\begin{array}{c}17.07 \mathrm{a} \\
15.06 \mathrm{bc} \\
16.54 \mathrm{ab} \\
15.96 \mathrm{a}-\mathrm{c}\end{array}$ & $\begin{array}{l}23.51 \mathrm{ab} \\
21.41 \mathrm{a}-\mathrm{c} \\
18.14 \mathrm{~b}-\mathrm{d} \\
17.17 \mathrm{~b}-\mathrm{d}\end{array}$ \\
\hline $\begin{array}{c}\text { Time means } \\
30 \\
21 \\
7\end{array}$ & & $\begin{array}{l}- \\
- \\
- \\
-\end{array}$ & $\begin{array}{l}1.45 \\
1.13 \\
1.72 \\
\end{array}$ & $\begin{array}{l}15.59 \\
15.76 \\
16.16 \\
\end{array}$ & $\begin{array}{l}18.81 \\
18.84 \\
20.06\end{array}$ \\
\hline 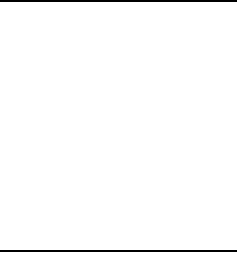 & $\begin{array}{c}\text { Dose means } \\
0 \\
100 \\
125 \\
150 \\
\end{array}$ & $\begin{array}{l}- \\
- \\
- \\
- \\
-\end{array}$ & $\begin{array}{l}2.87 \\
1.15 \\
1.17 \\
0.55\end{array}$ & $\begin{array}{l}16.40 \\
15.41 \\
15.68 \\
15.86 \\
\end{array}$ & $\begin{array}{l}26.35 \\
18.73 \\
17.43 \\
14.43 \\
\end{array}$ \\
\hline $\begin{array}{c}P \text { values } \\
\text { Time }(\mathrm{T}) \\
\text { AVG Dose }(\mathrm{D}) \\
\text { T x D }\end{array}$ & & $\begin{array}{l}- \\
- \\
- \\
-\end{array}$ & $\begin{array}{l}0.441 \\
0.166 \\
0.000\end{array}$ & $\begin{array}{l}0.427 \\
0.218 \\
0.049\end{array}$ & $\begin{array}{l}0.851 \\
0.181 \\
0.001\end{array}$ \\
\hline
\end{tabular}

${ }^{1}$ Days before harvest (DBH)

*Each column, values followed by the same letter are not significantly different at $p<0.05$ level according to Duncan's multiple range test

\section{Fruit and leave mineral element contents determination}

The effect of AVG applications on the nitrogen contents of fruits was found to be statistically significant $(\mathrm{p}<0.05)$ in both years as time $\mathrm{x}$ dose interaction. In both years, nitrogen contents increased with 30 and $21 \mathrm{DBH}$ AVG applications but decreased with 7 DBH AVG applications. The highest and lowest nitrogen contents were obtained from 30 DBH 150 ppm AVG and from 7 DBH 125 ppm AVG, respectively (Table 7).

Table 7. The effect of AVG treatments on nitrogen, calcium, magnesium in the 'Akça' pear fruit, 2012 and 2013

\begin{tabular}{c|c|c|c|c|c|c|c}
\hline \multirow{2}{*}{ Application time $^{1}$} & \multirow{2}{*}{$\begin{array}{c}\text { AVG doses } \\
(\mathbf{p p m})\end{array}$} & \multicolumn{2}{|c|}{ Nitrogen (\%) } & \multicolumn{2}{c|}{ Calcium (ppm) } & \multicolumn{2}{c}{ Magnesium (ppm) } \\
\cline { 3 - 8 } & & I. Year & II. Year & I. Year & II. Year & I. Year & II. Year \\
\hline \multirow{3}{*}{$30 \mathrm{~d}$} & 0 & $0.50 \mathrm{bc} *$ & $0.52 \mathrm{a}^{*}$ & 0.08 & $0.07 \mathrm{a}-\mathrm{c}^{*}$ & 0.06 & 0.06 \\
& 100 & $0.56 \mathrm{~b}$ & $0.57 \mathrm{ab}$ & 0.09 & $0.09 \mathrm{ab}$ & 0.07 & 0.07 \\
& 125 & $0.54 \mathrm{bc}$ & $0.55 \mathrm{ab}$ & 0.08 & $0.07 \mathrm{a}-\mathrm{c}$ & 0.06 & 0.06 \\
& 150 & $0.72 \mathrm{a}$ & $0.72 \mathrm{a}$ & 0.09 & $0.09 \mathrm{a}$ & 0.07 & 0.07 \\
\hline \multirow{3}{*}{$21 \mathrm{~d}$} & 0 & $0.39 \mathrm{c}$ & $0.50 \mathrm{~b}$ & 0.07 & $0.06 \mathrm{c}$ & 0.06 & 0.06 \\
& 100 & $0.55 \mathrm{~b}$ & $0.56 \mathrm{ab}$ & 0.10 & $0.09 \mathrm{a}$ & 0.06 & 0.06 \\
& 125 & $0.51 \mathrm{bc}$ & $0.50 \mathrm{~b}$ & 0.05 & $0.07 \mathrm{bc}$ & 0.05 & 0.05 \\
& 150 & $0.55 \mathrm{bc}$ & $0.55 \mathrm{ab}$ & 0.09 & $0.09 \mathrm{a}$ & 0.07 & 0.07 \\
\hline \multirow{3}{*}{$7 \mathrm{~d}$} & 0 & $0.50 \mathrm{bc}$ & $0.48 \mathrm{~b}$ & 0.07 & $0.06 \mathrm{c}$ & 0.05 & 0.07 \\
& 100 & $0.42 \mathrm{bc}$ & $0.42 \mathrm{~b}$ & 0.08 & $0.08 \mathrm{abc}$ & 0.05 & 0.05 \\
& 125 & $0.41 \mathrm{bc}$ & $0.41 \mathrm{~b}$ & 0.08 & $0.08 \mathrm{abc}$ & 0.06 & 0.06 \\
& 150 & $0.46 \mathrm{bc}$ & $0.46 \mathrm{~b}$ & 0.08 & $0.08 \mathrm{abc}$ & 0.06 & 0.06 \\
\hline
\end{tabular}




$$
-5561 \text { - }
$$

\begin{tabular}{c|c|c|c|c|c|c|c}
\hline Time means & & & & & & & \\
30 & & 0.58 & 0.59 & 0.08 & 0.08 & 0.06 & 0.07 \\
21 & & 0.50 & 0.53 & 0.08 & 0.08 & 0.06 & 0.06 \\
7 & & 0.45 & 0.44 & 0.08 & 0.08 & 0.06 & 0.06 \\
\hline & Dose means & & & & & & \\
& 0 & 0.46 & 0.50 & $0.07 \mathrm{bc}$ & 0.07 & 0.06 & 0.06 \\
& 100 & 0.51 & 0.52 & $0.09 \mathrm{ab}$ & 0.09 & 0.06 & 0.06 \\
& 125 & 0.49 & 0.49 & $0.07 \mathrm{c}$ & 0.07 & 0.06 & 0.06 \\
& 150 & 0.57 & 0.58 & $0.09 \mathrm{a}$ & 0.09 & 0.07 & 0.07 \\
\hline$P$ values & & & & & & & \\
Time (T) & & 0.008 & 0.002 & 0.732 & 0.683 & 0.155 & 0.237 \\
AVG Dose (D) & & 0.154 & 0.347 & 0.025 & 0.000 & 0.175 & 0.144 \\
T x D & & 0.004 & 0.041 & 0.195 & 0.030 & 0.239 & 0.187 \\
\hline
\end{tabular}

${ }^{1}$ Days before harvest (DBH)

*Each column, values followed by the same letter are not significantly different at $p<0.05$ level according to Duncan's multiple range test

In the variance analysis made for calcium content, only the time $\mathrm{x}$ dose interaction of the applications in the second year was found statistically significant $(\mathrm{p}<0.05)$. In general, calcium content increased with the applications. In terms of magnesium content, however, no statistically significant difference was observed in both years (Table 7). In terms of phosphorus and potassium, there was no statistically significant difference in phosphorus content in both years, while the difference between dose averages was statistically significant $(\mathrm{p}<0.05)$ in potassium content and the dose of 150 ppm had the highest amount of potassium (Table 8).

Table 8. The effect of AVG treatments on phosphorus, potassium, copper, zinc in the 'Akça' pear fruit, 2012 and 2013

\begin{tabular}{c|c|c|c|c|c|c|c|c|c}
\hline \multirow{2}{*}{$\begin{array}{c}\text { Application } \\
\text { time }\end{array}$} & $\begin{array}{c}\text { AVG doses } \\
\text { (ppm) }\end{array}$ & \multicolumn{2}{|c|}{$\begin{array}{c}\text { Phosphorus } \\
(\mathbf{p p m})\end{array}$} & \multicolumn{2}{c|}{$\begin{array}{c}\text { Potassium } \\
\text { (ppm) }\end{array}$} & \multicolumn{2}{c|}{ Copper (ppm) } & \multicolumn{2}{c}{ Zinc (ppm) } \\
\cline { 3 - 10 } & & I. Year & II. Year & I. Year & II. Year & I. Year & $\begin{array}{c}\text { II. } \\
\text { Year }\end{array}$ & $\begin{array}{c}\text { I. } \\
\text { Year }\end{array}$ & $\begin{array}{c}\text { II. } \\
\text { Year }\end{array}$ \\
\hline \multirow{3}{*}{$30 \mathrm{~d}$} & 0 & 0.12 & 0.12 & 1.18 & 1.18 & 1.82 & 1.83 & $9.17 \mathrm{~b}^{*}$ & 9.41 \\
& 100 & 0.13 & 0.14 & 1.21 & 1.22 & 3.65 & 3.76 & $12.64 \mathrm{a}$ & 12.80 \\
& 125 & 0.11 & 0.11 & 1.04 & 1.04 & 2.28 & 2.15 & $11.28 \mathrm{ab}$ & 12.29 \\
& 150 & 0.14 & 0.14 & 1.28 & 1.29 & 2.68 & 3.10 & $13.92 \mathrm{a}$ & 14.08 \\
\hline \multirow{3}{*}{$21 \mathrm{~d}$} & 0 & 0.12 & 0.12 & 1.16 & 1.12 & 1.74 & 2.44 & $9.09 \mathrm{~b}$ & 9.42 \\
& 100 & 0.12 & 0.12 & 1.14 & 1.14 & 2.30 & 2.30 & $11.25 \mathrm{ab}$ & 11.81 \\
& 125 & 0.08 & 0.08 & 0.79 & 0.79 & 3.08 & 2.98 & $10.90 \mathrm{ab}$ & 11.20 \\
& 150 & 0.13 & 0.13 & 1.21 & 1.22 & 3.31 & 3.21 & $10.95 \mathrm{ab}$ & 10.97 \\
\hline \multirow{3}{*}{$7 \mathrm{~d}$} & 0 & 0.12 & 0.12 & 1.16 & 1.12 & 1.67 & 2.63 & $9.14 \mathrm{~b}$ & 9.21 \\
& 100 & 0.11 & 0.11 & 1.01 & 1.01 & 2.44 & 2.61 & $8.25 \mathrm{~b}$ & 8.17 \\
& 125 & 0.11 & 0.12 & 1.03 & 1.04 & 2.56 & 2.66 & $8.85 \mathrm{~b}$ & 9.26 \\
& 150 & 0.12 & 0.12 & 1.07 & 1.12 & 2.93 & 3.10 & $10.85 \mathrm{ab}$ & 10.98 \\
\hline
\end{tabular}




\begin{tabular}{c|c|c|c|c|c|c|c|c|c}
\hline $\begin{array}{c}\text { Time means } \\
30\end{array}$ & & 0.13 & 0.13 & 1.18 & 1.18 & 2.61 & 2.71 & 11.75 & $12.15 \mathrm{a}^{*}$ \\
21 & & 0.11 & 0.11 & 1.08 & 1.07 & 2.61 & 2.73 & 10.55 & $10.85 \mathrm{ab}$ \\
7 & & 0.11 & 0.12 & 1.07 & 1.07 & 2.40 & 2.75 & 9.27 & $9.40 \mathrm{~b}$ \\
\hline & Dose means & & & & & & & & \\
& 0 & 0.12 & 0.12 & $1.17 \mathrm{a}^{*}$ & $1.14 \mathrm{a}^{*}$ & 1.74 & 2.30 & 9.13 & 9.34 \\
& 100 & 0.12 & 0.12 & $1.12 \mathrm{a}$ & $1.12 \mathrm{a}$ & 2.79 & 2.89 & 10.72 & 10.93 \\
& 125 & 0.10 & 0.10 & $0.95 \mathrm{~b}$ & $0.96 \mathrm{~b}$ & 2.64 & 2.60 & 10.34 & 10.92 \\
& 150 & 0.13 & 0.13 & $1.18 \mathrm{a}$ & $1.21 \mathrm{a}$ & 2.97 & 3.14 & 11.91 & 12.01 \\
\hline$P$ values & & & & & & & & & \\
Time (T) & & 0.331 & 0.335 & 0.260 & 0.219 & 0.882 & 0.995 & 0.016 & 0.016 \\
AVG Dose (D) & & 0.107 & 0.110 & 0.017 & 0.019 & 0.101 & 0.300 & 0.051 & 0.130 \\
T x D & & 0.272 & 0.276 & 0.059 & 0.078 & 0.596 & 0.608 & 0.015 & 0.055 \\
\hline
\end{tabular}

${ }^{1}$ Days before harvest $(\mathrm{DBH})$

*Each column, values followed by the same letter are not significantly different at $p<0.05$ level according to Duncan's multiple range test

In both years, the effect of AVG applications on iron content was statistically significant $(\mathrm{p}<0.05)$ as time $\mathrm{x}$ dose interaction. However, manganese and boron contents were not found to be statistically significant (Table 9). The effect of AVG applications on copper and zinc contents is given in Table 8. In zinc content, the time $\mathrm{x}$ dose interaction was statistically significant $(p<0.05)$ only in the first year of AVG applications. The application with the highest iron content was $30 \mathrm{DBH} 150 \mathrm{ppm}$ AVG in both years. On the other hand, the lowest iron content was observed in the control fruits with 7 DBH AVG (Table 9).

Table 9. The effect of AVG treatments on iron, manganese, boron in the 'Akça' pear fruit, 2012 and 2013

\begin{tabular}{c|c|c|c|c|c|c|c}
\hline \multirow{2}{*}{ Application time $^{1}$} & AVG doses & \multicolumn{2}{|c|}{ Iron (ppm) } & \multicolumn{2}{c|}{ Manganese (ppm) } & \multicolumn{2}{c}{ Boron (ppm) } \\
\cline { 3 - 8 } & $(\mathbf{p p m})$ & I. Year & II. Year & I. Year & II. Year & I. Year & II. Year \\
\hline \multirow{3}{*}{$30 \mathrm{~d}$} & 0 & $13.90 \mathrm{e}^{*}$ & $14.45 \mathrm{~cd} *$ & 5.27 & 5.61 & 12.00 & 11.48 \\
& 100 & $19.27 \mathrm{ab}$ & $18.91 \mathrm{ab}$ & 7.23 & 7.73 & 17.73 & 17.99 \\
& 125 & $17.33 \mathrm{~b}-\mathrm{d}$ & $17.37 \mathrm{~b}-\mathrm{d}$ & 6.14 & 6.41 & 11.97 & 12.03 \\
& 150 & $21.21 \mathrm{a}$ & $21.22 \mathrm{a}$ & 7.07 & 7.20 & 14.71 & 15.36 \\
\hline \multirow{3}{*}{$21 \mathrm{~d}$} & 0 & $13.87 \mathrm{e}$ & $13.82 \mathrm{~d}$ & 4.84 & 5.32 & 12.14 & 12.71 \\
& 100 & $17.04 \mathrm{~b}-\mathrm{e}$ & $17.09 \mathrm{~b}-\mathrm{d}$ & 6.60 & 6.78 & 12.41 & 12.28 \\
& 125 & $17.78 \mathrm{bc}$ & $17.75 \mathrm{bc}$ & 6.24 & 6.57 & 13.02 & 12.60 \\
& 150 & $17.01 \mathrm{~b}-\mathrm{e}$ & $16.86 \mathrm{~b}-\mathrm{d}$ & 6.11 & 6.55 & 13.39 & 13.46 \\
\hline \multirow{3}{*}{$7 \mathrm{~d}$} & 0 & $14.03 \mathrm{e}$ & $14.09 \mathrm{~cd}$ & 4.75 & 5.26 & 12.60 & 11.19 \\
& 100 & $14.45 \mathrm{de}$ & $14.91 \mathrm{~cd}$ & 5.40 & 5.74 & 15.21 & 15.51 \\
& 125 & $14.98 \mathrm{c}-\mathrm{e}$ & $14.96 \mathrm{~cd}$ & 5.87 & 6.09 & 14.90 & 15.18 \\
Time means & 150 & $16.37 \mathrm{~b}-\mathrm{e}$ & $16.21 \mathrm{~b}-\mathrm{d}$ & 5.67 & 6.00 & 11.82 & 13.40 \\
\hline 30 & & & & & & & \\
21 & & 17.93 & 17.99 & 6.43 & 6.74 & 14.10 & 14.21 \\
7 & & 16.43 & 16.38 & 5.95 & 6.30 & 12.74 & 12.76 \\
& & 14.96 & 15.04 & 5.42 & 5.77 & 13.63 & 13.82 \\
\hline
\end{tabular}




\begin{tabular}{c|c|c|c|c|c|c|c}
\hline & Dose means & & & & & & \\
& 0 & 13.93 & 14.12 & 4.95 & 5.40 & 12.25 & 11.79 \\
& 100 & 16.92 & 16.97 & 6.41 & 6.75 & 15.12 & 15.26 \\
& 125 & 16.70 & 16.69 & 6.08 & 6.36 & 13.30 & 13.27 \\
& 150 & 18.20 & 18.10 & 6.28 & 6.58 & 13.31 & 14.07 \\
\hline$P$ values & & & & & & & \\
Time (T) & & 0.019 & 0.019 & 0.159 & 0.107 & 0.625 & 0.582 \\
AVG Dose (D) & & 0.003 & 0.007 & 0.055 & 0.042 & 0.361 & 0.191 \\
T x D & & 0.000 & 0.002 & 0.310 & 0.171 & 0.659 & 0.453 \\
\hline
\end{tabular}

${ }^{1}$ Days before harvest (DBH)

*Each column, values followed by the same letter are not significantly different at $p<0.05$ level according to Duncan's multiple range test

The effect of AVG applications was found to be statistically insignificant on the nitrogen content of leaves but statistically significant $(\mathrm{p}<0.05)$ on the magnesium content as time $\mathrm{x}$ dose interaction only in the second year and again statistically significant $(\mathrm{p}<0.05)$ on the calcium content as time $\mathrm{x}$ dose interaction in both years of the project (Table 10).

Table 10. The effect of AVG treatments on nitrogen, calcium, magnesium in the 'Akça' pear leave, 2012 and 2013

\begin{tabular}{|c|c|c|c|c|c|c|c|}
\hline \multirow{2}{*}{ Application time ${ }^{1}$} & \multirow{2}{*}{$\begin{array}{c}\text { AVG doses } \\
(\mathbf{p p m})\end{array}$} & \multicolumn{2}{|c|}{ Nitrogen (\%) } & \multicolumn{2}{|c|}{ Calcium (ppm) } & \multicolumn{2}{|c|}{ Magnesium (ppm) } \\
\hline & & I. Year & II. Year & I. Year & II. Year & I. Year & II. Year \\
\hline \multirow{4}{*}{$30 \mathrm{~d}$} & 0 & 1.98 & 1.92 & $1.98 b^{*}$ & $1.80 \mathrm{~b}^{*}$ & 0.43 & $0.47 \mathrm{ab}^{*}$ \\
\hline & 100 & 1.95 & 1.82 & $1.78 \mathrm{bc}$ & $1.55 \mathrm{~cd}$ & 0.40 & $0.39 \mathrm{c}$ \\
\hline & 125 & 2.07 & 1.99 & $2.03 \mathrm{ab}$ & $2.06 \mathrm{a}$ & 0.44 & $0.48 \mathrm{a}$ \\
\hline & 150 & 2.05 & 1.85 & $1.95 \mathrm{bc}$ & $1.64 \mathrm{~b}-\mathrm{d}$ & 0.40 & $0.39 \mathrm{c}$ \\
\hline \multirow{4}{*}{$21 \mathrm{~d}$} & 0 & 1.93 & 1.79 & $1.79 \mathrm{bc}$ & $1.82 \mathrm{~b}$ & 0.42 & $0.48 \mathrm{a}$ \\
\hline & 100 & 1.97 & 1.89 & $2.42 \mathrm{a}$ & $1.76 b c$ & 0.42 & $0.43 \mathrm{a}-\mathrm{c}$ \\
\hline & 125 & 1.95 & 1.91 & $2.03 \mathrm{ab}$ & $1.81 \mathrm{~b}$ & 0.43 & $0.46 \mathrm{ab}$ \\
\hline & 150 & 2.02 & 1.89 & $1.76 \mathrm{bc}$ & $1.72 \mathrm{~b}-\mathrm{d}$ & 0.41 & $0.45 \mathrm{ab}$ \\
\hline \multirow{4}{*}{$7 \mathrm{~d}$} & 0 & 2.00 & 1.76 & $1.50 \mathrm{c}$ & $1.78 b c$ & 0.25 & $0.45 \mathrm{ab}$ \\
\hline & 100 & 2.03 & 1.95 & $1.89 \mathrm{bc}$ & $1.51 \mathrm{~d}$ & 0.42 & $0.43 \mathrm{a}-\mathrm{c}$ \\
\hline & 125 & 2.04 & 1.83 & $1.97 \mathrm{bc}$ & $1.60 \mathrm{~b}-\mathrm{d}$ & 0.40 & $0.42 b c$ \\
\hline & 150 & 1.96 & 1.90 & $1.92 \mathrm{bc}$ & $1.83 \mathrm{~b}$ & 0.43 & $0.46 \mathrm{ab}$ \\
\hline \multicolumn{8}{|l|}{ Time } \\
\hline 30 & & 2.01 & 1.89 & 1.94 & 1.76 & 0.42 & 0.43 \\
\hline 21 & & 1.97 & 1.87 & 2.00 & 1.78 & 0.42 & 0.46 \\
\hline \multirow[t]{6}{*}{7} & & 2.01 & 1.86 & 1.82 & 1.68 & 0.38 & 0.44 \\
\hline & Dose means & & & & & & \\
\hline & 0 & 1.97 & 1.83 & 1.75 & 1.80 & 0.37 & 0.47 \\
\hline & 100 & 1.98 & 1.89 & 2.03 & 1.61 & 0.41 & 0.42 \\
\hline & 125 & 2.02 & 1.91 & 2.01 & 1.82 & 0.43 & 0.45 \\
\hline & 150 & 2.01 & 1.88 & 1.88 & 1.73 & 0.41 & 0.44 \\
\hline$P$ values & & & & & & & \\
\hline Time $(\mathrm{T})$ & & 0.262 & 0.818 & 0.322 & 0.352 & 0.296 & 0.299 \\
\hline AVG Dose (D) & & 0.498 & 0.549 & 0.152 & 0.037 & 0.404 & 0.033 \\
\hline$x D$ & & 0.349 & 0.609 & 0.034 & 0.001 & 0.161 & 0.009 \\
\hline
\end{tabular}

${ }^{1}$ Days before harvest (DBH)

*Each column, values followed by the same letter are not significantly different at $p<0.05$ level according to Duncan's multiple range test 
The leaves with the highest calcium content $(2.42 \mathrm{ppm})$ were obtained from $21 \mathrm{DBH}$ $100 \mathrm{ppm}$ AVG in the first year and from $30 \mathrm{DBH} 125 \mathrm{ppm}$ AVG in the second year (Table 10). In terms of magnesium contents, the amount of magnesium decreased with the applications in the second year in general; it increased only in $30 \mathrm{DBH} 125 \mathrm{ppm}$ AVG (Table 10). With respect to the effect of AVG applications on phosphorus and potassium contents, the time $\mathrm{x}$ dose interaction for phosphorus and the difference between doses for potassium were found statistically significant $(\mathrm{p}<0.05)$ (Table 11). Table 11 shows the effect of AVG applications on the copper and zinc contents of the Akça leaves. It was seen that the applications increased the copper content. The effect of AVG applications was statistically significant on the iron and boron contents of leaves as well. As time $\mathrm{x}$ dose interaction, iron content in both years and boron content only in the second year were found statistically significant $(\mathrm{p}<0.05)$ (Table 12). Iron content had the highest value in $30 \mathrm{DBH} 125 \mathrm{ppm}$ AVG in the first year and in $7 \mathrm{DBH} 100 \mathrm{ppm}$ AVG in the second year. For boron, however, it was 7 DBH 100 ppm AVG with a value of $36.72 \mathrm{ppm}$ (Table 12).

Table 11. The effect of AVG treatments on phosphorus, potassium, copper, zinc in the 'Akça' pear leave, 2012 and 2013

\begin{tabular}{|c|c|c|c|c|c|c|c|c|c|}
\hline \multirow{2}{*}{$\begin{array}{l}\text { Application } \\
\text { time }^{1}\end{array}$} & \multirow{2}{*}{$\begin{array}{l}\text { AVG doses } \\
\quad(\text { ppm) }\end{array}$} & \multicolumn{2}{|c|}{$\begin{array}{l}\text { Phosphorus } \\
\text { (ppm) }\end{array}$} & \multicolumn{2}{|c|}{ Potassium (ppm) } & \multicolumn{2}{|c|}{ Copper (ppm) } & \multicolumn{2}{|c|}{ Zinc (ppm) } \\
\hline & & $\begin{array}{l}\text { I. } \\
\text { Year }\end{array}$ & $\begin{array}{l}\text { II. } \\
\text { Year }\end{array}$ & $\begin{array}{l}\text { I. } \\
\text { Year }\end{array}$ & $\begin{array}{l}\text { II. } \\
\text { Year }\end{array}$ & $\begin{array}{l}\text { I. } \\
\text { Year }\end{array}$ & $\begin{array}{l}\text { II. } \\
\text { Year }\end{array}$ & $\begin{array}{l}\text { I. } \\
\text { Year }\end{array}$ & $\begin{array}{l}\text { II. } \\
\text { Year }\end{array}$ \\
\hline \multirow{4}{*}{$30 \mathrm{~d}$} & 0 & $0.19 \mathrm{a}-\mathrm{d}^{*}$ & 0.16 & 1.04 & 1.32 & $10.25 d^{*}$ & 9.61 & 34.91 & 36.74 \\
\hline & 100 & $0.17 b-d$ & 0.17 & 1.18 & 1.37 & $14.25 \mathrm{ab}$ & 12.86 & 40.40 & 35.99 \\
\hline & 125 & $0.18 b-d$ & 0.16 & 1.32 & 1.30 & $12.89 \mathrm{a}-\mathrm{d}$ & 13.55 & 38.71 & 33.40 \\
\hline & 150 & $0.16 \mathrm{c}$ & 0.16 & 1.34 & 1.28 & $12.83 \mathrm{a}-\mathrm{d}$ & 10.26 & 31.70 & 29.81 \\
\hline \multirow{4}{*}{$21 \mathrm{~d}$} & 0 & $0.22 \mathrm{a}$ & 0.16 & 1.04 & 1.21 & $10.49 \mathrm{~cd}$ & 9.37 & 35.80 & 34.58 \\
\hline & 100 & $0.21 \mathrm{a}-\mathrm{c}$ & 0.15 & 1.36 & 1.25 & $14.76 \mathrm{ab}$ & 9.76 & 36.06 & 28.35 \\
\hline & 125 & $0.17 b-d$ & 0.16 & 1.23 & 1.26 & 13.64ab & 12.13 & 39.73 & 30.51 \\
\hline & 150 & $0.16 c$ & 0.15 & 1.09 & 1.33 & $15.33 \mathrm{a}$ & 9.65 & 34.82 & 31.23 \\
\hline \multirow{4}{*}{$7 \mathrm{~d}$} & 0 & $0.21 \mathrm{ab}$ & 0.15 & 0.97 & 1.24 & $10.13 \mathrm{~d}$ & 9.65 & 36.60 & 35.22 \\
\hline & 100 & $0.16 c$ & 0.15 & 1.16 & 1.31 & $13.30 \mathrm{a}-\mathrm{c}$ & 15.04 & 33.82 & 31.84 \\
\hline & 125 & $0.16 \mathrm{c}$ & 0.15 & 1.15 & 1.45 & $11.98 \mathrm{~b}-\mathrm{d}$ & 11.34 & 37.22 & 31.19 \\
\hline & 150 & $0.18 b-d$ & 0.16 & 1.04 & 1.41 & $12.58 \mathrm{a}-\mathrm{d}$ & 12.55 & 37.79 & 34.03 \\
\hline \multicolumn{10}{|l|}{ Time means } \\
\hline 30 & & 0.17 & 0.16 & 1.22 & 1.32 & 12.56 & 11.57 & 36.43 & 33.99 \\
\hline 21 & & 0.19 & 0.15 & 1.18 & 1.26 & 13.56 & 10.23 & 36.60 & 31.17 \\
\hline \multirow{5}{*}{ 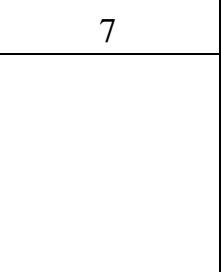 } & & 0.18 & 0.16 & 1.08 & 1.35 & 12.00 & 12.15 & 36.36 & 33.07 \\
\hline & Dose means & & & & & 1029 & 954 & 3577 & 3552 \\
\hline & 100 & 0.18 & $\begin{array}{l}0.16 \\
0.16\end{array}$ & $\begin{array}{r}1.02 \mathrm{~b}^{*} \\
1.23 \mathrm{a}\end{array}$ & $\begin{array}{l}1.20 \\
1.31\end{array}$ & $\begin{array}{l}10.29 \\
14.11\end{array}$ & 12.55 & 36.76 & 32.06 \\
\hline & 125 & 0.17 & 0.16 & $1.24 \mathrm{a}$ & 1.34 & 12.84 & 12.34 & 38.56 & 31.70 \\
\hline & 150 & 0.17 & 0.16 & $1.15 \mathrm{ab}$ & 1.34 & 13.58 & 10.82 & 34.77 & 31.69 \\
\hline$P$ values & & & & & & & & & \\
\hline Time $(\mathrm{T})$ & & 0.281 & 0.085 & 0.149 & 0.220 & 0.182 & 0.246 & 0.987 & 0.159 \\
\hline AVG Dose (D) & & 0.006 & 0.919 & 0.025 & 0.465 & 0.000 & 0.080 & 0.155 & 0.067 \\
\hline$T \times D$ & & 0.015 & 0.232 & 0.053 & 0.534 & 0.002 & 0.187 & 0.134 & 0.065 \\
\hline
\end{tabular}

${ }^{1}$ Days before harvest (DBH)

*each column, values followed by the same letter are not significantly different at $\mathrm{p}<0.05$ level according to Duncan's multiple range test 
Table 12. The effect of AVG treatments on iron, manganese, boron in the 'Akça' pear leave, 2012 and 2013

\begin{tabular}{|c|c|c|c|c|c|c|c|}
\hline \multirow{2}{*}{$\begin{array}{c}\text { Application } \\
\text { time }^{1}\end{array}$} & \multirow{2}{*}{$\begin{array}{l}\text { AVG doses } \\
\quad(p p m)\end{array}$} & \multicolumn{2}{|c|}{ Iron (ppm) } & \multicolumn{2}{|c|}{ Manganese (ppm) } & \multicolumn{2}{|c|}{ Boron (ppm) } \\
\hline & & I. Year & II. Year & I. Year & II. Year & I. Year & II. Year \\
\hline \multirow{4}{*}{$30 \mathrm{~d}$} & 0 & $62.37 \mathrm{ab} *$ & $59.36 \mathrm{ab} *$ & 57.25 & 176.43 & 32.03 & $32.84 \mathrm{bc} *$ \\
\hline & 100 & $67.46 \mathrm{a}$ & $51.82 \mathrm{c}-\mathrm{e}$ & 69.46 & 147.17 & 34.71 & $33.90 \mathrm{bc}$ \\
\hline & 125 & $71.00 \mathrm{a}$ & $58.83 \mathrm{a}-\mathrm{c}$ & 65.42 & 166.37 & 36.88 & $33.29 b c$ \\
\hline & 150 & $69.33 \mathrm{a}$ & 51.09de & 53.55 & 144.13 & 33.71 & $32.08 \mathrm{~cd}$ \\
\hline \multirow{4}{*}{$21 \mathrm{~d}$} & 0 & $54.19 b$ & $59.24 \mathrm{a}-\mathrm{c}$ & 56.79 & 158.95 & 31.70 & $32.75 b c$ \\
\hline & 100 & $67.19 \mathrm{a}$ & $52.48 b-e$ & 51.36 & 151.43 & 36.63 & 29.95de \\
\hline & 125 & $64.96 \mathrm{a}$ & $49.36 \mathrm{e}$ & 61.15 & 146.80 & 34.05 & $29.19 \mathrm{e}$ \\
\hline & 150 & $70.40 \mathrm{a}$ & $56.95 \mathrm{a}-\mathrm{d}$ & 65.22 & 148.10 & 37.70 & $35.41 \mathrm{ab}$ \\
\hline \multirow{4}{*}{$7 \mathrm{~d}$} & 0 & $62.17 \mathrm{ab}$ & $59.84 \mathrm{ab}$ & 59.66 & 139.71 & 31.12 & $32.86 b c$ \\
\hline & 100 & $69.39 \mathrm{a}$ & $61.71 \mathrm{a}$ & 72.77 & 175.83 & 35.55 & $36.72 \mathrm{a}$ \\
\hline & 125 & $69.07 \mathrm{a}$ & $57.34 \mathrm{a}-\mathrm{d}$ & 66.52 & 154.23 & 32.12 & $31.66 c-e$ \\
\hline & 150 & $64.49 a$ & $56.62 \mathrm{a}-\mathrm{d}$ & 60.85 & 158.60 & 31.66 & $32.97 \mathrm{bc}$ \\
\hline \multicolumn{8}{|l|}{ Time means } \\
\hline 30 & & 67.54 & 55.27 & 61.42 & 158.53 & 34.33 & 33.03 \\
\hline 21 & & 64.19 & 54.51 & 58.63 & 151.32 & 35.02 & 31.83 \\
\hline \multirow[t]{5}{*}{7} & & 66.28 & 58.88 & 64.95 & 157.09 & 32.62 & 33.55 \\
\hline & $\begin{array}{c}\text { Dose means } \\
0\end{array}$ & 59.58 & 59.48 & 57.90 & 158.36 & 31.62 & 32.82 \\
\hline & 100 & 68.01 & 55.33 & 64.53 & 158.14 & 35.63 & 33.52 \\
\hline & 125 & 68.34 & 55.17 & 64.37 & 155.80 & 34.35 & 31.38 \\
\hline & 150 & 68.07 & 54.89 & 59.87 & 150.28 & 34.36 & 33.49 \\
\hline \multicolumn{8}{|l|}{$P$ values } \\
\hline Time (T) & & 0.406 & 0.074 & 0.414 & 0.736 & 0.199 & 0.186 \\
\hline AVG Dose (D) & & 0.001 & 0.170 & 0.543 & 0.883 & 0.069 & 0.180 \\
\hline $\mathrm{T} \times \mathrm{D}$ & & 0.011 & 0.008 & 0.546 & 0.700 & 0.124 & 0.000 \\
\hline
\end{tabular}

${ }^{1}$ Days before harvest (DBH)

*Each column, values followed by the same letter are not significantly different at $p<0.05$ level according to Duncan's multiple range test

\section{The effect of applications on leaf area $\left(\mathrm{cm}^{2}\right)$ and chlorophyll value (spad value) in the leaves of 'Akça' variety}

The effect of AVG applications on the leaf area of 'Akça' variety was statistically significant $(\mathrm{p}<0.05)$ as time $\mathrm{x}$ dose interaction only in the first year. Leaf area expanded with the applications, and the largest leaf area was observed in $21 \mathrm{DBH}$ $125 \mathrm{ppm}$ AVG (Table 13). Their effect on the chlorophyll value of the leaves of 'Akça' variety was found statistically significant $(\mathrm{p}<0.05)$ as time $\mathrm{x}$ dose interaction in both years. The amount of chlorophyll increased in general with the applications. The highest spad value was obtained from $30 \mathrm{DBH} 125 \mathrm{ppm}$ AVG and 21 and 7 DBH $100 \mathrm{ppm}$ AVG (Table 13). 
Table 13. The effect of AVG treatments on Leaf area and total chlorophyll in the 'Akça' pear leave, 2012 and 2013

\begin{tabular}{|c|c|c|c|c|c|}
\hline \multirow{2}{*}{ Application time ${ }^{1}$} & \multirow{2}{*}{$\begin{array}{c}\text { AVG doses } \\
(\text { ppm) }\end{array}$} & \multicolumn{2}{|c|}{ Leaf area $\left(\mathrm{cm}^{2}\right)$} & \multicolumn{2}{|c|}{ Total chlorophyll (spad value) } \\
\hline & & I. Year & II. Year & I. Year & II. Year \\
\hline \multirow{4}{*}{$30 \mathrm{~d}$} & 0 & $33.01 \mathrm{~cd}^{*}$ & 34.65 & $44.12 b-d^{*}$ & $45.74 \mathrm{~cd}^{*}$ \\
\hline & 100 & $33.48 \mathrm{~cd}$ & 30.95 & $45.47 \mathrm{a}-\mathrm{c}$ & $46.78 b c$ \\
\hline & 125 & $36.32 \mathrm{~b}-\mathrm{d}$ & 36.73 & $46.07 \mathrm{ab}$ & $49.86 \mathrm{a}$ \\
\hline & 150 & $36.04 b-d$ & 39.45 & $44.07 \mathrm{~b}-\mathrm{d}$ & $47.74 a-c$ \\
\hline \multirow{4}{*}{$21 \mathrm{~d}$} & 0 & $32.33 d$ & 33.62 & $41.74 \mathrm{~d}$ & $44.11 \mathrm{~d}$ \\
\hline & 100 & $36.70 \mathrm{bc}$ & 36.23 & $48.16 \mathrm{a}$ & $49.60 \mathrm{a}$ \\
\hline & 125 & $41.01 \mathrm{a}$ & 38.41 & $44.87 \mathrm{a}-\mathrm{d}$ & $48.78 \mathrm{ab}$ \\
\hline & 150 & $36.17 b-d$ & 38.27 & $45.14 \mathrm{a}-\mathrm{d}$ & $47.92 \mathrm{a}-\mathrm{c}$ \\
\hline \multirow{4}{*}{$7 \mathrm{~d}$} & 0 & $33.11 \mathrm{~cd}$ & 32.21 & $42.95 b-d$ & $43.99 \mathrm{~d}$ \\
\hline & 100 & $34.50 \mathrm{~cd}$ & 34.57 & $46.13 \mathrm{ab}$ & $48.99 \mathrm{ab}$ \\
\hline & 125 & 38.96ab & 38.35 & $42.24 \mathrm{~cd}$ & 48.40ab \\
\hline & 150 & $39.56 \mathrm{ab}$ & 38.49 & $42.63 b-d$ & $48.30 \mathrm{ab}$ \\
\hline \multicolumn{6}{|l|}{ Time means } \\
\hline 30 & & 34.71 & 35.45 & 44.93 & 47.53 \\
\hline 21 & & 36.55 & 36.63 & 44.98 & 47.60 \\
\hline \multirow[t]{6}{*}{7} & & 36.53 & 35.90 & 43.49 & 47.42 \\
\hline & Dose means & & & & \\
\hline & 0 & 32.82 & 33.49 & 42.93 & 44.61 \\
\hline & 100 & 34.89 & 33.92 & 46.59 & 48.46 \\
\hline & 125 & 38.76 & 37.83 & 44.40 & 49.01 \\
\hline & 150 & 37.26 & 38.74 & 43.95 & 47.99 \\
\hline \multicolumn{6}{|l|}{$P$ values } \\
\hline Time $(\mathrm{T})$ & & 0.283 & 0.816 & 0.221 & 0.981 \\
\hline AVG Dose (D) & & 0.106 & 0.201 & 0.022 & 0.598 \\
\hline$T \times D$ & & 0.000 & 0.322 & 0.000 & 0.000 \\
\hline
\end{tabular}

${ }^{1}$ Days before harvest (DBH)

*Each column, values followed by the same letter are not significantly different at $p<0.05$ level according to Duncan's multiple range test

\section{Discussion}

It was determined that $30 \mathrm{DBH}$ and $21 \mathrm{DBH}$ AVG applications (all doses) delayed the harvest maturity of Akça pear for up to 3-4 days. It was well documented that AVG inhibits ethylene biosynthesis, thus retards fruit ripening (Amarante et al., 2002; Schupp and Greene, 2004; Petri et al., 2006; Kang et al., 2007). Similar results were also observed in this study. Clayton et al. (2000) reported that ReTain applied 14 and 7 days before harvest delayed the harvest for up to 4-10 days in the Bartlett pear. The increase in fruit weight with the AVG applications also led to an increase in fruit yield. As reported by Romani et al. (1983), since AVG allows for late ripening, fruit continues to grow, leading to an increase in size and yield. Delayed harvest provides flexibility in fruit processing-packing, storage and marketing planning. Pasa et al. (2017) stated that fruit set, number of fruit per tree and yield were significantly improved by AVG sprayed one week after full bloom with doses ranging from 40 to $100 \mathrm{mg} \mathrm{L}^{-1}$ for 'Santa 
Maria', while for 'Abate Fetel' the most responsive dose was $100 \mathrm{mg} \mathrm{L}^{-1}$. Fruit quality attributes and fruit size were not affected by treatments. Dussi et al. (2002) applied 14 DBH 125 and 180 ppm AVG to Williams pears. In this study, AVG did not have any effect on pre-harvest fall, but an increase in size and hardness was detected. Pre-harvest AVG applications to Williams pear caused a delay in fruit ripening and increased fruit size (Romani et al., 1983). In our study, compared to the control fruits, the AVG applications increased fruit width by about $2-16 \%$ and fruit weight by $7-57 \%$.

In the study performed by Clayton et al. (2000) on Bartlett pear, it is reported that AVG increased fruit firmness compared to the control group and maintained both their shelf life and storage as well as their firmness more than controls. The 'Akça' pear is in demand in both domestic and international markets because it is an early comer summer variety. Therefore, shelf life shortens during transport. Higher fruit firmness means a longer shelf life. For this reason, in line with the data obtained by this study, an increase in fruit firmness is considered to reduce quality losses during transport. According to our findings, $\mathrm{L}^{*}$ values expressing brightness decreased with the AVG applications. $\mathrm{a}^{*}$ values indicating redness were lower in the AVG applications, which is parallel with AVG's retardant effect on ripening and thus coloring.

All AVG applications slowed both ethylene production $\left(\mu \mathrm{L} \mathrm{kg}^{-1} \mathrm{~h}\right)$ and respiratory rate $\left(\mu \mathrm{L} \mathrm{kg}^{-1} \mathrm{~h}\right)$ in the fruit. Similar to our findings, Clayton et al. (2000) and Butar and Çetinbaş (2017) reported that AVG reduced both ethylene amount and respiration rate and that the application doses had the same effect in Williams pear. In the AVG studies conducted on other varieties, it was reported that ethylene production and respiration rate slowed down (Amarante et al., 2002; Schuup and Greene, 2004; Petri et al., 2006; Kang et al., 2007; Ozturk et al., 2012; Y1ld1z et al., 2012).

When applied externally, plant growth regulators, which are synthesized naturally in the plant, may have different physiological effects depending on application period and concentration (Salisbury and Ross, 1985). In our study, different application periods and doses of AVG plant growth regulator affected fruits' mineral element contents differently even in different years. Butar and Çetinbaş (2017) stated that a change in AVG application time and dose led to different mineral element contents in 'Williams' pear fruits. In a study conducted on the 'Akça' pear, the contents of $\mathrm{N}, \mathrm{P}, \mathrm{K}, \mathrm{Ca}, \mathrm{Mg}$, $\mathrm{Zn}, \mathrm{Fe}, \mathrm{Mn}$ and $\mathrm{Cu}$ in the fruit were found to be 3.37, 0.73, 8.25, 0.65, 8.25, 6.63, 4.08, $3.01 \mathrm{mg} \mathrm{kg}^{-1}$, respectively (Kuçuker et al., 2015a). Partly similar results were obtained in our study. AVG applications affect mineral element contents in different ways in leaves as in fruits. It is reported that pear contains $1.134-1.682 \%$ nitrogen, $0.46-1.08 \%$ potassium, $1.81-3.1 \%$ calcium, $0.28-0.55 \%$ magnesium, $0.078-0.22 \%$ phosphorus, 51.5 $197.5 \mathrm{mg} \mathrm{kg}^{-1}$ iron, 4.85-8.0 $\mathrm{mg} \mathrm{kg}^{-1}$ copper, 16-57.5 $\mathrm{mg} \mathrm{kg}^{-1}$ zinc, and $20-99.5 \mathrm{mg} \mathrm{kg}^{-1}$ manganese (Gunen et al., 2003). In our study, these elements were found to exist in the above-given ranges. AVG plant growth regulator is thought to have both increasing and decreasing effects on the mineral element contents in the fruits and leaves of 'Akça' pear. However, decreases did not negatively affect fruit or leaf quality in our study.

Since ethylene hormone causes chlorophyll decomposition in plants (Taiz and Zieger, 2008; Karaçalı, 2009), in an environment where ethylene hormone is suppressed, chlorophyll decomposition is either delayed or slowed down. Accordingly, in our opinion, the AVG applications in our study led to an increase in both leaf area and chlorophyll amount because delayed ethylene production enabled the leaves to nourish and photosynthesize more and have a larger area, and delayed chlorophyll decomposition gave rise to a higher chlorophyll amount. 


\section{Conclusion}

Taking all results into account, it was concluded in present study that AVG was definitely effective in increasing the quality of 'Akça' pears. The treatments applied in a dose of $100 \mathrm{ppm} 21$ days before the anticipated harvest time had more effective results in 'Akça' pear in terms of harvest time and fruit quality. Furthermore, since plant nutrients (macro and micro) and plant growth regulator are in interaction with each other, more detailed studies are necessary on this subject.

Acknowledgements. The author is thankful to Mr. Sinan Butar in the Fruit Research Institute, for help field management and chemical analysis.

\section{REFERENCES}

[1] Amarante, C. V. T., Anderson, S., Megguer, C. A., Blum, L. E. B. (2002): Effect of minoethoxyvinilglycine (AVG) on preharvest fruit drop and maturity of apples. Revista Brasileira de Fruticultura 24: 661-664.

[2] Andreotti, C., Bregoli, A. M., Costa, G. (2004): Pre- and post-harvest aminoethoxyvinylglycine (AVG) application affects maturity and storage of pear fruit. European Journal of Horticultural Science 69(4): 147-152.

[3] Barut, E. (1995): The use of plant growth regulator on horticulturae in future. - Derim 11: 141-144.

[4] Butar, S., Çetinbaş, M. (2017): Pre-Harvest application of Retain [aminoethoxyvinylglycine (AVG)] influences pre-harvest drop and fruit quality of 'Williams' pears. - Journal of Agricultural Sciences 23: 353-365.

[5] Clayton, M., Biasi, W. V., Southwick, S. M., Mitcham, E. J. (2000): ReTain affects maturity and ripening of 'Bartlett' pear. - HortScience 35(7): 1294-1299.

[6] Dilmaçunal, T. (2009): Normal and controlled atmosphere storage of some apple cultivars grown in organic and conventional farming conditions. - PhD Thesis, SDU Institute of Natural and Applied Sciences, Horticulture, Isparta, Turkey.

[7] Dussi, M. C., Sosa, D., Calvo, G. S. (2002): Effects of ReTain ${ }^{\mathrm{TM}}$ on fruit maturity and fruit set of pear cultivars Williams and Packham S Triumph. - Acta Horticulturae 596: 767-771.

[8] FAOSTAT (2018): http://www.faostat.fao.org. - Accessed in May 2018.

[9] Gunen, Y., Yagmur, B., Misirl1, A., Gulcan, R. (2003): Determination of mineral nutrients in some pear cultivars resistant and susceptible to fire blight. - Ege Journal of Agricultural Research 40(3): 65-72.

[10] Inada, K. (1963): Studies on a method for determining the deepness of green color and chlorophyll content of intact crop leaves and its practical applications principles for estimating the deepness of green color and chlorophyll content of whole leaves. Japanese Journal of Crop Science 32: 157-162.

[11] Jobling, J., Pradhan, R., Morris, S., Mitchell, L., Rath, A. (2003): The effect of ReTain ${ }^{\mathrm{TM}}$ plant growth regulator [aminoethoxyvinylglycine (AVG)] on the postharvest storage life of 'Tegan Blue' plums. - Australian Journal of Experimental Agriculture 43: 515-518.

[12] Kang, I. K., Byun, J. K., Kweon, H. J., Kim, M. J., Kwon, S., Park, M. Y., Lee, D. H., Choi, C., Choi, D. G. (2007): Effects of aminoethoxyvinylglycine on preharvest drop, fruit color, and quality of Tsugaru apples. - Horticulture, Environment, and Biotechnology 48(3): 159-164.

[13] Karaçal1, I. (2009): Conservation and Marketing of Horticultural Products (in Turkish). Ege University Agricultural Faculty, Bornova, Izmir, Turkey. 
[14] Kuçuker, E., Ozturk, B., Ozkan, Y., Yı1dız, K. (2015a): The effect of foliar urea treatment on yield, fruit quality and bioactive compounds of different pear varieties (Pyrus communis L.). - Ömer Halisdemir University Engineering Sciences Journal 4(2): 78-86.

[15] Kuçuker, E., Ozturk, B., Aksit, H., Genç, N. (2015b): Effect of pre-harvest aminoethoxyvinylglycine (avg) application on bioactive compounds and fruit quality of plum (Prunus salicina lindell cv. black beauty) at the time of harvest and during cold storage. - The Journal of Animal \& Plant Sciences 25(3): 763-770.

[16] Kucukyumuk, C., Sarısu, H. C., Yıldız, H., Kaçal, E., Koçal, H. (2015): Farklı anaçlar üzerine aşılı 0900 Ziraat Kiraz çeşidinde su stresinin bazı vejetatif gelişim parametrelerine etkisi. - Yüzüncü Y1l University Journal Agriculture Science 25(2): 180192.

[17] Lurie, S. (2008): Regulation of ethylene biosynthesis in fruits by aminoethoxyvinylglycine and 1-methylcyclopropene. - Acta Horticulturae 796: 31-41.

[18] Martinez-Romero, D., Bailen, G., Serrano, M., Guillén, F., Valverde, J. M., Zapata, P., Castillo, S., Valero, D. (2007): Tools to maintain postharvest fruit and vegetable quality through the inhibition of ethylene action: a review. - Critical Reviews in Food Science and Nutrition 47: 543-560.

[19] Ozelkok, S., Kaynaş, K., Buyukyılmaz, M. (1995): Investigate the physiology of the possible types of important arms foreseen in production. - Scientific Research and Review 48.

[20] Ozturk, B., Kuçuker, E., Karaman, S., Ozkan, Y. (2012): The effects of cold storage and aminoethoxyvinylgcine (AVG) on bioactive compounds of plum fruit (Prunus salicina Lindell cv. 'Black Amber'). - Postharvest Biology and Technology 72: 35-41.

[21] Pasa, M. S., Silva, C. P., Carra, B., Brighenti, A. F., Souza, A. L. K., Schmitz, J. D., Katsurayama, J. M., Ciotta, M. N. (2017): Fruit set and yield of 'Santa Maria' and 'Abate Fetel' pears are increased by early spring application of aminoethoxyvinilglycine (AVG). - Revista de Ciências Agroveterinárias, Lages 16(4): 487-491.

[22] Pech, J. C., Purgatto, E., Bouzayen, M., Latche, A. (2012): Ethylene and fruit ripening. Annual Plant Reviews 44: 275-304.

[23] Petri, J. L., Leite, G. B., Argenta, L. C., Basso, C. (2006): Ripening delay and fruit drop control in 'Imperial Gala' and 'Suprema' ('Fuji' sport) apples by applying AVG (aminoethoxyvinylglycine). - Acta Horticulturae 727: 519-524.

[24] Rath, A. C., Prentice, A. J. (2004): Yield increase and higher flesh firmness of 'Arctic Snow' nectaries both at harvest in Australia and after export to Taiwan following preharvest application of retain plant growth regulator (aminoethoxyvinylglycine, AVG). Australian Journal of Experimental Agriculture 44: 343-351.

[25] Romani, R., Labavitch, J., Yamashita, T., Hess, B., Rae, H. (1983): Preharvest AVG treatment of 'Bartlett' pear fruits: Effects on ripening, color change, and 104 volatiles. Journal of the American Society for Horticultural Science 108(6): 1046-1049.

[26] Ryan, J., Estafan, G., Rashid, A. (2001): Soil and Plant Analysis Laboratory Manual. $2^{\text {nd }}$ ed. - ICARDA and NARS, Aleppo, Syria, pp. 135-140.

[27] Salisbury, F. B., Ross, C. W. (1985): Plant Physiology. 3rd ed. - Wadsworth, Belmont, California.

[28] Schupp, J. R., Greene, D. W. (2004): Effect of aminoethoxyvinylglycine (AVG) on preharvest drop, fruit quality, and maturation of 'McIntosh' apples. I. Concentration and timing of dilute applications of AVG. - HortScience 39: 1030-1035.

[29] Taiz, L., Zieger, E. (2008): Plant Physiology (in Turkish translation, editor: İsmail Turkan). - Palme Publishing, Ankara, Turkey.

[30] Yildiz, K., Ozturk, B., Ozkan, Y. (2012): Effects aminoethoxyvinylglycine (AVG) on preharvest fruit drop, fruit maturity, and quality of 'Red Chief' apple. - Scientia Horticulturae 144: 121-124. 